\title{
Manejo de la ruptura hepática espontánea en el síndrome de HELLP
}

\author{
Management of spontaneous hepatic rupture in HELLP syndrome
}

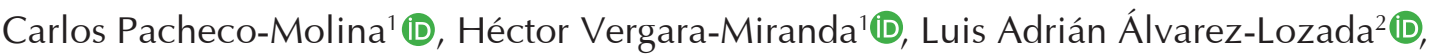 \\ Francisco Vásquez-Fernández ${ }^{1}$
}

1 Médico, especialista en Cirugía General, Hospital Universitario Dr. José Eleuterio González, Universidad Autónoma de Nuevo León, Monterrey, México.

2 Estudiante de medicina, Grupo estudiantil con interés en el campo quirúrgico, Universidad Autónoma de Nuevo León, Monterrey, México.

\section{Resumen}

Introducción. El síndrome de HELLP es una variante grave de la preeclampsia, con una baja incidencia, entre el 0,5-0,9\% de todos los embarazos. La ruptura hepática espontánea en el embarazo es infrecuente, presenta una alta mortalidad, y se ha relacionado con hemangiomas hepáticos, coriocarcinoma y síndrome de HELLP.

Caso clínico. Mujer de 29 años con embarazo de 34,3 semanas, con actividad uterina progresiva, sufrimiento fetal agudo y hallazgos compatibles con síndrome de HELLP. Se practicó cesárea de urgencia encontrando hemoperitoneo y ruptura hepática del lóbulo derecho, por lo que se realizó empaquetamiento abdominal y posterior reintervención quirúrgica.

Discusión. La ruptura hepática espontánea en el embarazo es una entidad infrecuente y potencialmente mortal. Es esencial un alto nivel de sospecha para realizar el diagnóstico y la rápida intervención. Requiere un manejo multidisciplinario para un resultado exitoso. Se han descrito múltiples tratamientos que dependen de las manifestaciones clínicas y extensión de la lesión, pero es claro que la laparotomía primaria y el taponamiento constituyen la mejor elección ante el hallazgo intraoperatorio.

Palabras clave: síndrome de HELLP; rotura espontánea; hígado; complicaciones del embarazo; cirugía; reintervención; control de daños.

\footnotetext{
Abstract

Introduction. HELLP syndrome is a severe variant of preeclampsia with a low incidence, between $0.5-0.9 \%$ of all pregnancies. Spontaneous hepatic rupture in pregnancy is rare, has a high mortality, and has been associated with hepatic hemangiomas, choriocarcinoma, and HELLP syndrome.

Fecha de recibido: 21/05/2020 - Fecha de aceptación: 20/08/2020 - Fecha de publicación en línea: 12/04/2021

Correspondencia: Carlos Pacheco-Molina, Hospital Universitario Dr. José Eleuterio González, S/N, Col. Mitras Centro, C.P. 64460, Monterrey, México. Teléfono: +52 818389 1111. Correo electrónico: pacheco.mc45@gmail.com

Citar como: Pacheco-Molina C, Vergara-Miranda H, Álvarez-Lozada LA, Vásquez-Fernández F. Manejo de la ruptura hepática espontánea en el síndrome de HELLP. Rev Colomb Cir. 2021;36:549-53. https://doi.org/10.30944/20117582.664

Este es un artículo de acceso abierto bajo una Licencia Creative Commons - BY-NC-ND https://creativecommons.org/licenses/by-ncnd/4.0/deed.es
} 
Clinical case. 29-year-old woman with a 34.3-week pregnancy, with progressive uterine activity and acute fetal distress and findings compatible with HELLP syndrome. An emergency cesarean section was performed, finding hemoperitoneum and hepatic rupture of the right lobe, performing abdominal packing and subsequent surgical reoperation.

Discussion. Spontaneous liver rupture in pregnancy is a rare and potentially fatal entity. A high level of suspicion is essential to make the diagnosis and prompt intervention. It requires multidisciplinary management for a successful outcome. Multiple treatments have been described that depend on the clinical manifestations and extent of the lesion, but it is clear that primary laparotomy and packing constitute the best choice before the intraoperative finding.

Keywords: HELLP syndrome; rupture, spontaneous; liver pregnancy complications; surgery reoperation; damage control.

\section{Introducción}

La ruptura hepática espontanea en el embarazo es una condición infrecuente, que conlleva a una alta mortalidad materna y perinatal. Por lo general, se asocia con hemangiomas hepáticos, metástasis de coriocarcinoma o con síndrome de HELLP (por las siglas: H para hemólisis, EL para enzimas hepáticas elevadas y LP para plaquetas bajas, de su denominación en inglés) ${ }^{1,2}$. Su aparición súbita y su resultado potencialmente fatal lo convierten en un importante desafío diagnóstico y terapéutico.

Acorde a lo reportado por Escobar, la ruptura hepática se presenta generalmente en mujeres alrededor de los 40 años, multíparas, dentro de las primeras 15 horas postparto, generalmente después de las 32 semanas de gestación, asociado a congestión intravascular hepática por depósitos de fibrina, así como a vasoespasmo por la alta concentración de vasopresores durante el embarazo ${ }^{3}$.

El síndrome HELLP es una variante grave de la preeclampsia, que se caracteriza por una tríada de hemólisis, enzimas hepáticas elevadas y trombocitopenia, y tiene una baja incidencia $(0,17-0,9 \% \text { de todos los embarazos })^{4,5}$. Cuando se presenta después del parto, generalmente, se asocia con una mayor tasa de morbilidad y mortalidad materna, por un mayor riesgo de desarrollar edema pulmonar, insuficiencia renal, coagulación intravascular diseminada y hematoma subcapsular hepático ${ }^{6}$.

El objetivo de este artículo fue presentar el caso de una mujer embarazada llevada a cesárea de emergencia por diagnóstico de síndrome de
HELLP, que presentó una ruptura hepática, y fue tratada exitosamente. Se enfatiza la necesidad del diagnóstico temprano, el tratamiento quirúrgico oportuno y el seguimiento posoperatorio de los pacientes en la unidad de cuidados intensivos.

\section{Presentación del caso}

Paciente femenina de 29 años, con antecedente de dos embarazos normales, cursando con su tercer embarazo, de 34,3 semanas de gestación y adecuado control prenatal, quien acudió al servicio de Urgencias Obstétricas por actividad uterina progresiva de más de siete horas de evolución. A su ingreso se encontró hemodinámicamente estable, sin respuesta inflamatoria, con fondo uterino de $32 \mathrm{~cm}$, feto único vivo, situación longitudinal, presentación cefálica, dorso izquierdo, y tacto vaginal con cérvix blando, dilatación de $2 \mathrm{~cm}$, sin salida de líquido.

Debido a que el registro cardiotocográfico inicial fue clase 1 , se inició útero inhibición, pero posteriormente se encontraron datos de sufrimiento fetal agudo, por lo que se decidió realizar cesárea de urgencia, en la cual se encontró un hemoperitoneo de 300 cc. Se realizó histerotomía tipo Kerr, obteniendo un neonato masculino prematuro, y se exploró la cavidad abdominal, con hallazgo de una lesión hepática. Con apoyo del Cirugía General se procedió a la ampliar la herida quirúrgica encontrando un hematoma subcapsular hepático, abierto a cavidad abdominal (figura 1), que comprometía los segmentos V, VI, VII y VIII (figura 2). Se efectuó taponamiento abdominal con 17 compresas y cierre de cavidad abdominal. 


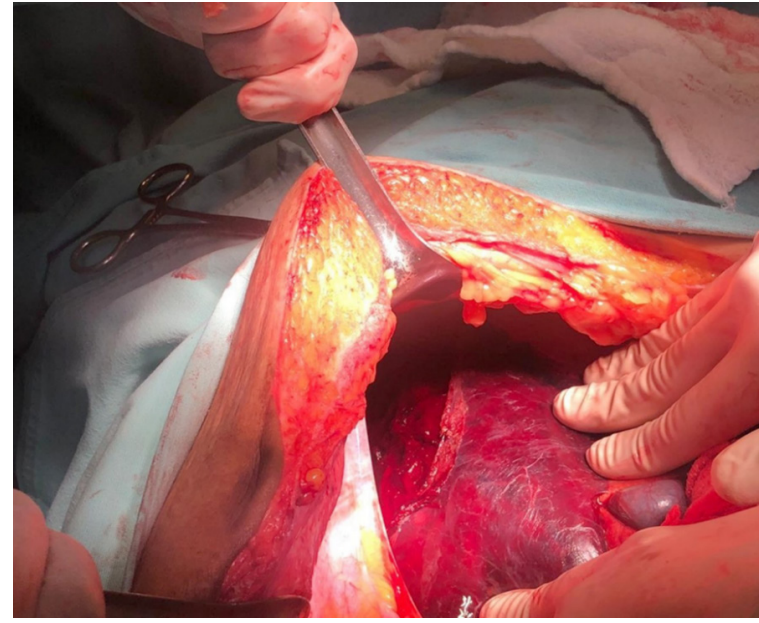

Figura 1. Laparotomía donde se aprecia la ruptura hepática del lóbulo derecho.

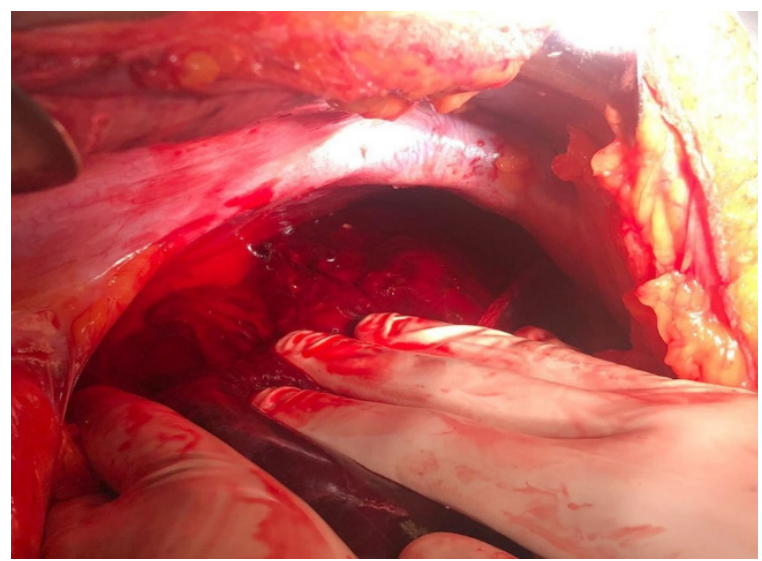

Figura 2. Ruptura hepática de los segmentos VII - VIII

Requirió manejo posoperatorio en la unidad de cuidados intensivos, con intubación orotraqueal y soporte ventilatorio, control antihipertensivo con nitroglicerina, impregnación con sulfato de magnesio para prevención de eclampsia y transfusión de hemoderivados. Presentó una adecuada evolución y a las 48 horas se realizó un segundo tiempo quirúrgico con desempaquetamiento, y hallazgos de líquido seroso peritoneal, sin sangrado activo (figura 3). Se verificó la hemostasia de los segmentos hepáticos, se dejó un agente hemostático absorbible y se instalaron drenajes cerrados. En el posoperatorio evolucionó adecuadamente,

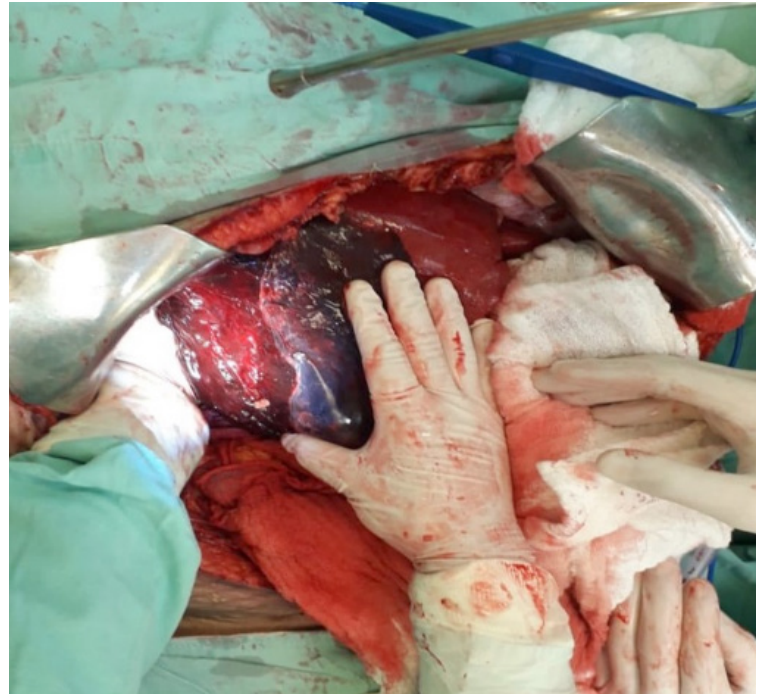

Figura 3. Desempaquetamiento del lóbulo hepático derecho

con control de las cifras de presión arterial y laboratorios séricos con disminución de enzimas hepáticas, por lo cual se dio el alta médica.

\section{Discusión}

La ruptura hepática espontánea en el embarazo, que fue descrita por primera vez por Abercrombie en 1984, es una condición rara, con una incidencia que varía de 1 en 40,000 a 1 en 250,000 partos, con una alta mortalidad materna y perinatal ${ }^{5,7}$. Generalmente se asocia con preeclampsia, eclampsia o síndrome de HELLP, que presenta mayor riesgo de morbilidad y mortalidad materno fetal, que según se ha descrito en la literatura puede ser tan alta como de un $39-80 \%{ }^{3,8}$.

La mayoría de los casos de síndrome de HELLP se desarrollan antes del parto, entre las semanas 27-34 de gestación, y parecen ser la manifestación final de la lesión, que se caracteriza por daño endotelial microvascular y agregación plaquetaria intravascular, pero se desconoce el mecanismo exacto que conduce a la ruptura hepática ${ }^{9}$. Se ha mencionado como una probable causa la congestión intravascular hepática por depósitos de fibrina y el vasoespasmo inducido por la alta concentración de vasopresores durante el embarazo ${ }^{3}$. 
Los síntomas y signos de sangrado hepático que se presentan son inespecíficos, como dolor en el cuadrante superior derecho, epigastralgia, náusea y vómito. Por lo tanto, es necesaria una alta sospecha clínica cuando una mujer embarazada presenta repentinamente un dolor abdominal intenso e inexplicable ${ }^{3,5,10}$.

Actualmente son de gran utilidad diagnóstica el ultrasonido y la tomografía computarizada ${ }^{11}$. Particularmente, el ultrasonido permite detectar lesiones hepáticas antes de su presentación clínica súbita ${ }^{7}$. En el caso de esta paciente, y debido a la urgencia quirúrgica, no fue posible la realización de estudios diagnósticos por imagen. El diagnóstico de esta entidad casi siempre se realiza intraoperatoriamente durante la cesárea o en el posparto inmediato.

De acuerdo con la revisión de la literatura, la exploración y el tratamiento quirúrgico de la lesión es el procedimiento más realizado, como ocurrió en nuestra paciente, en quien el empaquetamiento permitió el control de la hemorragia, evitó la aparición de la coagulación intravascular diseminada y el deterioro del estado hemodinámico. Las 48 horas de espera para la realización del segundo tiempo quirúrgico, representaron un tiempo valioso en el que se compensó la pérdida sanguínea y se mejoró el estado general de la paciente. El tratamiento realizado evitó tomar decisiones precipitadas, lo que finalmente resulto en la preservación de la funcionalidad hepática.

En la literatura se han informado distintos enfoques como el manejo conservador, la ligadura de la arteria hepática, embolización arterial, hepatorrafia, empaquetamiento hepático, uso de esponjas de colágeno, malla absorbible, pegamento de fibrina, coagulación con láser de argón, factor recombinante VIIa, e incluso, procedimientos más complejos como la resección hepática parcial o el trasplante hepático ${ }^{12-14}$. La tasa de supervivencia en el trasplante hepático y la embolización arterial puede ser de hasta el $100 \%$, pero depende de las características de la lesión al momento del abordaje ${ }^{14}$. La aplicación de estas alternativas depende en su totalidad de la experiencia del cirujano, la presentación clínica, la sospecha clínica y su detección oportuna, para brindar el mejor manejo disponible. En consecuencia, no todos los tratamientos son apropiados para todas las pacientes.

Dada la poca experiencia que se tiene con estos casos, el valor de esta publicación radica en el beneficio demostrado cuando se realiza una cirugía de control de daños, y su reintervención a las 48 horas, logrando un procedimiento menos mórbido y radical. El trasplante hepático no fue considerado porque en las condiciones clínicas de la paciente, lo primordial era solamente contener la hemorragia y lograr un estado hemodinámico óptimo. La resección lobar podría haber sido una alternativa viable en la resolución de la patología, sin embargo, en el segundo tiempo quirúrgico se observó que la afectación hepática era subcapsular, sin comprometer el sistema biliar, además de no presentar otras condiciones que ameritaran la resección del lóbulo. La embolización por radio-intervencionismo no fue contemplada dado que fue un hallazgo transoperatorio que requería su resolución en el momento.

Como lo sustenta el artículo de Vigil "La laparotomía primaria y el taponamiento de la fuente de sangrado no deben ser pospuestos y deben ser realizados en el hospital de primer nivel de atención, si el paciente no está suficientemente estable para ser transportado" ${ }^{14}$.

\section{Conclusiones}

La ruptura hepática espontánea en el embarazo es rara pero potencialmente mortal. Es fundamental conocer la patología y su presentación para sospechar el diagnóstico y establecer el abordaje de manera oportuna, con un bajo riesgo de mortalidad. Es necesario un enfoque multidisciplinario e integral.

La reintervención o segundo tiempo quirúrgico permiten la toma de decisiones importantes y definitivas para la resolución terapéutica. Siempre que se presente un paciente quirúrgico, hemodinámicamente inestable, y cuando las condiciones de la patología lo permitan, se debe considerar esta opción terapéutica. 


\section{Cumplimiento de normas éticas}

Consentimiento informado: los autores declaran que han seguido los protocolos de sus centros de trabajo sobre la publicación de datos de pacientes y se ha obtenido el consentimiento informado de la paciente. Este documento se encuentra en poder del autor de correspondencia.

Conflicto de interés: los autores declaran que no existen conflictos de interés.

Fuente de financiación: estudio de investigación no sujeto a financiamiento externo.

\section{Contribución de los autores:}

Concepción y diseño del estudio: Carlos Pacheco-Molina, Héctor Vergara-Miranda, Luis Adrián Álvarez-Lozada, Francisco Vásquez-Fernández.

Adquisición de datos: Carlos Pacheco-Molina, Héctor Vergara-Miranda, Luis Adrián Álvarez-Lozada, Francisco Vásquez-Fernández.

Análisis e interpretación de datos: Carlos Pacheco-Molina, Héctor Vergara-Miranda, Luis Adrián Álvarez-Lozada, Francisco Vásquez-Fernández.

Redacción del manuscrito: Carlos Pacheco-Molina, Héctor Vergara-Miranda, Luis Adrián Álvarez-Lozada, Francisco Vásquez-Fernández.

Revisión crítica: Carlos Pacheco-Molina, Héctor VergaraMiranda, Luis Adrián Álvarez-Lozada, Francisco VásquezFernández.

\section{Referencias}

1 Zhou X, Zhang M, Liu Z, Duan M, Dong L. A rare case of spontaneous hepatic rupture in a pregnant woman. BMC Pregnancy Childbirth. 2018;18:87. https://doi.org/10.1186/s12884-018-1713-5

2 Poo JL, Gongora J. Hepatic hematoma and hepatic rupture in pregnancy. Annals of Hepatology. 2006;5:224-6. https://doi.org/10.1016/S1665-2681(19)32017-4

3 Escobar-Vidarte M. F., Montes D., Pérez A., Loaiza-Osorio S., Nieto-Calvache A. J. Hepatic rupture associated with preeclampsia, report of three cases and literature review. The Journal of Maternal-Fetal \& Neonatal Medicine 32;16:2767-73.

https://doi.org/10.1080/14767058.2018.1446209

4 Troja A, Abdou A, Rapp C, Wienand S, Malik E, Raab HR. Management of spontaneous hepatic rupture on top of HELLP syndrome: case report and review of the literature. Viszeralmedizin. 2015;31:205-8. https://doi.org/10.1159/000376601

5 Cruz-Santiago J., Meza-Jiménez G., Ayala-López E.A., Velázquez-García J.A., Moreno-Ley P.I., Robledo-Meléndez A., et al., Ruptura hepática en el síndrome de HELLP, revisión del tratamiento quirúrgico. Cirujano General. 2020;42:31-7. https://doi.org/10.35366/92709

6 Celik C, Gezginc K, Altintepe L, Tonbul HZ, Yaman ST, Akyürek C. Results of the pregnancies with HELLP syndrome. Ren Fail. 2003;25:613-8. https://doi.org/10.1081/JDI-120022553

7 Wicke C, Pereira PL, Neeser E. Subcapsular liver hematoma in HELLP syndrome: evaluation of diagnostic and therapeutic options - a unicenter study. Am J Obstet Gynecol. 2004;190:106-12. https://doi.org/10.1016/j.ajog.2003.08.029

8 Sibai BM, Ramadan MK, Usta I, Salama M, Mercer BM, Friedman SA. Maternal morbidity and mortality in 442 pregnancies with hemolysis, elevated liver enzymes, and low platelets (HELLP syndrome). Am J Obstet Gynecol. 1993;169:1000-6. https://doi.org/10.1016/0002-9378(93)90043-I

9 Pavlis T, Aloizos S, Aravosita P, Mystakelli C. Diagnosis and surgical management of spontaneous hepatic rupture associated with HELLP syndrome. J Surg Educ. 2009;66:163-7.

https://doi.org/10.1016/j.jsurg.2009.04.001

10 Mascarenhas R, Mathias J, Varadarajan R, Geoghegan J, Traynor O. Spontaneous hepatic rupture: a report of five cases. HPB (Oxford). 2002;4:167-70. https://doi.org/10.1080/13651820260503819

11 Rinehart BK, Terrone DA, Magann EF, Martin RW, May WL, Martin Jr JN. Preeclampsia-associated hepatic hemorrhage and rupture: mode of management related to maternal and perinatal outcome. Obstet Gynecol Surv. 1999;54:196-202. https://doi.org/10.1097/00006254-199903000-00024

12 Schwartz ML, Lien JM. Spontaneous liver hematoma in pregnancy not clearly associated with preeclampsia: a case presentation and literature review. Am J Obstet Gynecol. 1997;176:1328-32. https://doi.org/10.1016/S0002-9378(97)70353-3

13 Frise CJ, Davis P, Barker G, Wilkinson D, Mackillop L. Hepatic capsular rupture in pregnancy. Obstet Med. 2016;9:185-8. https://doi.org/10.1177/1753495X16670480

14 Vigil-De Gracia P, Ortega-Paz L. Pre-eclampsia/ eclampsia and hepatic rupture. Int J Gynaecol Obstet. 2012;118:186-9.

https://doi.org/10.1016/j.ijgo.2012.03.042 